\title{
Colorectal Lesions among Sudanese Patient Attending for Endoscopy-multi Center Study
}

\author{
Ashwag Mohammed Mukhtar ${ }^{1}$, Tarig Mohamed Hussein ${ }^{2}$, Amin abbas ${ }^{3}$, Muataz \\ SalahEIdin Abdelaziz ${ }^{2}$, Nassir Alhaboob ${ }^{4}$, ElSagad Eltayeb Gady ${ }^{5}$, Lamyaa \\ EIHassan $^{6}$
}

${ }^{1}$ Department of Histopathology and Cytology, AlNeelain University, Faculty of Medical Laboratory Sciences, Khartoum-Sudan. ${ }^{2}$ Department of Gastroenterology Surgery, Ibn Sina specialized Hospital, Khartoum, Sudan. ${ }^{3}$ Ibn Sina specialized Hospital, Khartoum, Sudan. ${ }^{4}$ Faculty of Medicine, Omdurman Islamic University, Department of GI Surgery, Ibn Sina Specialized Hospital, Khartoum, Sudan. ${ }^{5}$ Faculty of Medicine, Al Neelain University, Department of GI Surgery, Ibn Sina Specialized Hospital, Khartoum, Sudan. ${ }^{6}$ Faculty of Medicine, Al Ahfad University for women, Khartoum, Sudan.

\begin{abstract}
Background: Colorectal cancer is one of the most significant causes of cancer death. With records of high incidence and late detection of the disease in Sudan, and it was found to affect younger age groups. Methods: This study aimed to explore the incidence of colorectal lesions in the main endoscopic centers in Khartoum state- Sudan. It was a cross-sectional prospective study; data were collected from patients attending the main endoscopic centers in Sudan- Khartoum state during February 2013 to June 2014 including Khartoum teaching hospital, Neelain medical diagnostic center and Ibn sina hospital. Results: One hundred and six participants were diagnosed endoscopically with colorectal lesions, 44 (41.5\%) showed inflammatory mucosa, 40 (37.7\%) showed tumor and $22(20.7 \%)$ had polyps. In the whole group of patients the mean age was 45.7 years, $34.2 \%$ of tumor patients were less than 50 years old, $46.8 \%$ of the lesions were in the rectum, the highest frequency of tumor patients group were from western tribes, and the lesions were located commonly in the rectum. Conclusion: We concluded that the rectum is the most common site of cancer within the Sudanese population than other parts of the colon with apparently high incidence within young ages.
\end{abstract}

Keywords: Colorectal cancer- Endoscopy- Colorectal lesions

\section{Introduction}

Colorectal cancer $(\mathrm{CRC})$ is the third cancer in the world regarding incidence, and it is one of the most common malignancy in developed regions [1-2].

There is a large geographic difference in the global distribution of colorectal cancer. The developed world accounts for over $63 \%$ of all cases [3].

In Sudan, colorectal carcinoma is the most common malignancy of the Gastro-Intestinal tract [4]. And there is no statistical data regarding the geographical and racial distribution of the colonic tumors [5]. Few studies was conducted and revealed that there is a different disease pattern in Sudan with high incidence rates tending to affect younger populations than those internationally reported. The first presentation of the disease ranging from relatively healthy patient with few symptoms to life-threatening bowel obstruction with risk of perforation and fecal peritonitis [5].

According to recent studies more than $90 \%$ of colorectal cancer cases occur in people aged 50 or older. [6,7-8] However, it appears to be increasing among younger persons [9]. And in the United States it is now commonly diagnosed among men and women aged 20 to 49 years [10-12].

The progress of a colorectal cancer is thought to occur

Corresponding Author:

Dr. Ashwag Mohammed Mukhtar

Department of Histopathology and Cytology, AlNeelain University, Faculty of Medical Laboratory Sciences, Khartoum-Sudan.

Email: ashwagmmukhtar@yahoo.com 
in stages. The abnormal colonocytes with certain genetic alterations are able to proliferate excessively and acquire additional mutations, as this process continues the cells assume malignant characteristics of invasiveness and metastatic potential [13].

\section{Materials and Methods}

It was a cross-sectional prospective multi-center study targeting Gastro intestinal tract endoscopic centers in Khartoum state including Khartoum teaching hospital, Ibn Sina Hospital, Neelain medical Diagnostic center, during the period February 2013 to June 2014. Patients who attend the colonoscopy unit was randomly selected for the questionnaire which designed to collect data including endoscopic findings, age, gender, residence, geographic distribution, family history of colorectal cancer and the anatomical sites of the lesions.

All the participants signed an informed consent about their agreement to participate in the study, and the ethical approval was taken from the ethical approval board of AlNeelain University.

\section{Results}

One hundred and six participants were diagnosed endoscopically with colorectal lesions, 44 (41.5\%) showed inflammatory mucosa, 40 (37.7\%) showed tumor and 22 (20.7\%) had polyps (Figure 1).

Among the whole group of patients $63.2 \%$ (67) of the cases were male and $36.8 \%$ (39) were females.

The mean age was 45.7 years; the minimum was 7 years and the maximum 86 years old.

$60 \%$ of patients are living outside of Khartoum state and the geographic distribution of patients was 35.4\% from the west, $34.1 \%$ from central regions, $28 \%$ from the north and from each east and south tribes $1.2 \%$ as shown in (Figure 2).

Eight patients from the total 106 patients had family history of colorectal cancer and the pearson chi square test showed that there was a significant statistical relation (p. value $>0.05$ ) between the geographic distribution of

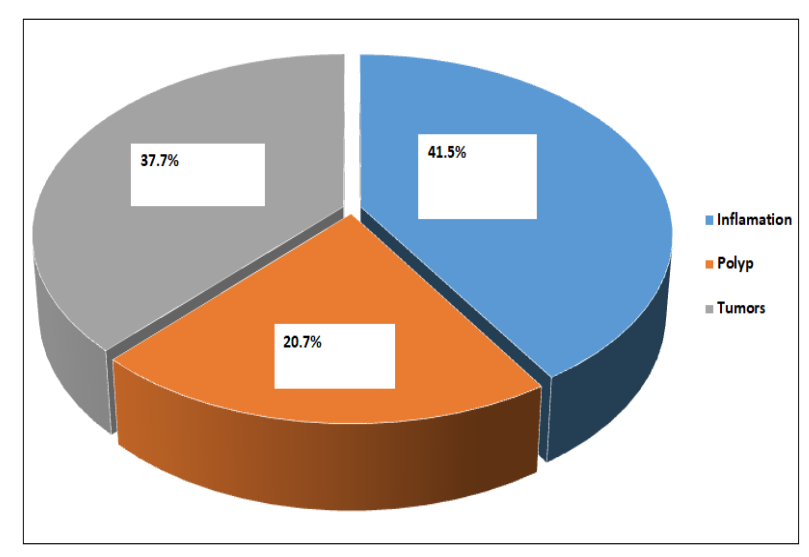

Figure 1. Frequencies of Endoscopic Colorectal Lesions. Showing the frequency of endoscopic lesions 44 (41.5\%) showed inflammatory mucosa, $40 \quad(37.7 \%)$ showed tumor and $22(20.7 \%)$ had polyps.

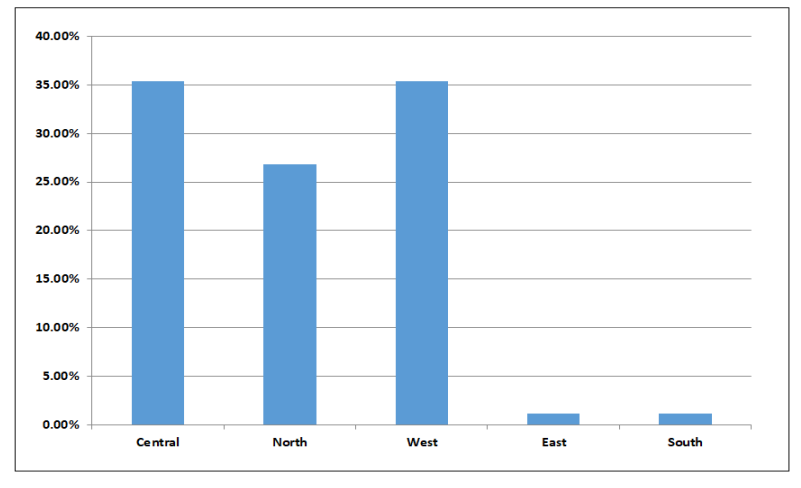

Figure 2. Geographic Distribution of the Sudanese Tribes among Patients. Geographic distribution of patients was $35.4 \%$ from the west, $34.1 \%$ from central regions, $28 \%$ from the north and from each east and south tribes $1.2 \%$.

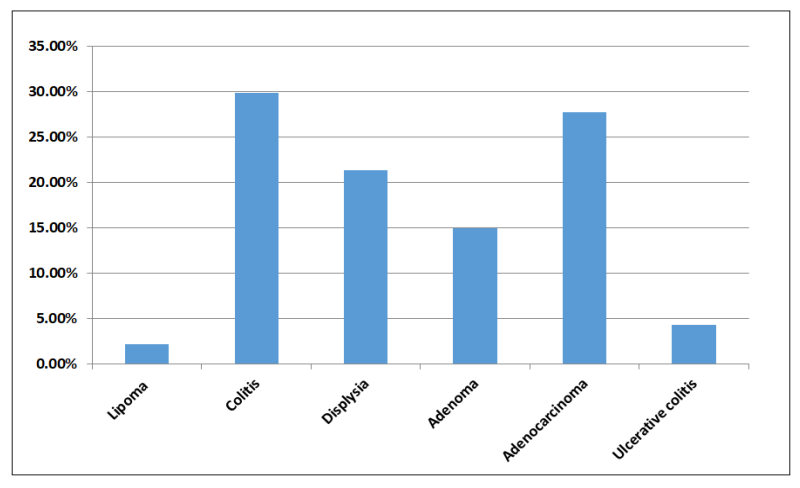

Figure 3. Frequencies of Histopathological Diagnosis. Showing the histopathology diagnosis of seventy-four samples, 14 samples $(29.8 \%)$ diagnosed with colitis, 13 (27.7\%) diagnosed with Adenocarcinoma, 10 (21.3\%) showed Dysplasia, and Adenoma in 7 samples (14.9\%), lipoma were in one sample $(2.1 \%)$ and ulcerative colitis in two $(4.3 \%)$.

patients and the family history of the colorectal cancer with a p. value (0.02).

The histopathology reports of seventy-four samples were got to confirm the diagnosis, 14 samples $(29.8 \%)$ diagnosed with colitis, $13(27.7 \%)$ diagnosed with Adenocarcinoma, 10 (21.3\%) showed Dysplasia, and Adenoma in 7 samples $(14.9 \%)$, lipoma were in one sample $(2.1 \%)$ and ulcerative colitis in two $(4.3 \%)$ shown in (Figure 3).

The cross tabulation with Chi square test showed a significant statistical relation between the diagnosis of endoscopy and the histopathological reports with p. value (0.00).

The endoscopic lesions were found within different anatomical parts of the large bowl; $46.8 \%$ were in the rectum, $41.5 \%$ were in the colon, $5.3 \%$ in the sigmoid, and $3.2 \%$ were in each cecum and anus. Using the pearson chi square test there was a significant statistical relation with a $p$ value $(0.00)$ between the site of lesion and the endoscopic diagnosis.

From the total of 40 patients diagnosed with tumor mass in colonoscopy $25(62.5 \%)$ were male and 15 $(37.5 \%)$ were females, 2 of them $(5.4 \%)$ had family 
history of colorectal cancer.

The geographic distribution of patients was 39.4\% from the west tribes followed by the northern and central tribes. $50 \%$ of the tumors were diagnosed with Adenocarcinoma in the confirmation with histopathology and $30 \%$ diagnosed with Dysplasia $.84 \%$ of the tumor masses were located in the rectum and 2 samples (5.3\%) were located in each sigmoid, cecum and colon.

Twenty two patients showed polypoid lesions endoscopically; $16(72.7 \%)$ of them were male and 6 $(27.3 \%)$ of them were female. Those who had family history with the colorectal cancer were $22.7 \%$ and $77.3 \%$ had no family history. The geographic distribution of this group was $40 \%$ from the west tribes and the rest $30 \%$ from central and north tribes equally, The histopathological diagnosis showed $46.2 \%$ have adenoma, $23.1 \%$ Dysplasia, $15.4 \%$ colitis and $7.7 \%$ diagnosed with adenocarcinoma. The higher frequency of polypoid lesions was $50 \%$ located in the colon, followed by the rectum $27.3 \%$, $13.6 \%$ sigmoid and $4.5 \%$ in the cecum From the total 22 patients who had polypoid lesions five of them had family history of colorectal cancer, the Chi square test showed statistical significant relation in the family history with the cancer between the healthy control group and the polypoid lesions group with p. value 0.01 , OR: $0.7, \mathrm{CI}$ 95\% 0.61-0.96). Within the 44 patients diagnosed with inflammatory lesions, $26(59.1 \%)$ were male and 18 (40.1\%) were female.

Regarding the anatomical location of the lesions $73.5 \%$ were in the colon and the lowest frequencies (5.9\%) were within the anus. $71.4 \%$ showed colitis in confirmation with the histopathology. The highest frequency of geographic distribution (55.2\%) was among the central tribes.

\section{Discussion}

The mean age of patients diagnosed with having tumor was 52.4; and $26.3 \%$ of them were less than 40 years old; this is comparable with a previous study performed in Ibn Sina Hospital at 2015, which found that colorectal cancer among young $(<40$ years old $)$ is $17.81 \%$, and $43.84 \%$ was below 50 years [14].

In Khartoum Teaching Hospital during the period $1^{\text {st }}$ January 2000 to $31^{\text {st }}$ December 2006; more than $34.5 \%$ of the study population was below the age of 40 years, and $17.3 \%$ were below 30 years. The peak frequency was at the age group (51-60) followed by the age group (61-70) [15].

In our study $34.2 \%$ of the tumor patients were less than 50 years old, and among the whole study groups the frequency of participants with mutated APC gene who were less than 55years old was $73.9 \%$ including tumor patients and others considered with high risk of cancer by having inflammatory mucosa or polypoid lesions.

The highest frequency of age among tumor patients was within the 50-60 age group, and in the polypoid lesions were within the $30-40$ years old, in the inflammatory group they were within the $40-50$ years age group, this indicates that the first onset of cancer begins in old ages, and the polyps and inflammatory lesions begin within young ages, that was also indicated by the noticed frequencies of young ages ( $>40$ year) among the different study groups which was, $26.3 \%$ among tumor group, $34.2 \%$ in inflammatory lesions group, and 63.6\% among polypoied lesions group .

The detection of cancer and the precursor lesions at early and more treatable stages is important for cure and survival. And it is previously known that the neoplastic polyps and inflammatory lesions are precursor lesions of the colorectal cancer. and nearly $95 \%$ of sporadic type of the cancer is developing from adenomas, and an individual with a history of adenomas has an increased risk of developing colorectal cancer, than individuals with no previous history of adenomas [7].

Regarding the anatomical site of lesions $84.2 \%$ of tumors were in the rectum this is agreed with Abdalla, et al. who found that rectal cancer represented half of the study population and rectal carcinomas are more common in men than in women [15] but disagree with who found that Colonic cancer constitutes $60.27 \%$ of the cases and rectal cancer found in $39.73 \%$ of cases [14].

Our findings revealed that $58.7 \%$ of patients were from outside of Khartoum this raises up the importance of improving health care centers in rural areas to avoid the late detection of cancers

and delay of treatment, although this was in disagreement with another study which found that the highest frequency were for those who are resident in Khartoum followed by western tribes [14].

Our findings revealed that the western tribes were the highest frequency of geographic distribution in the tumor and polyps group but within the inflammation group the central tribes were the highest, this can indicate the type of diet or the mode of cancer within these different tribes, and the genetic factors.

Eight of the participants showed having history of colorectal cancer in their family within the first degree relatives, $62.5 \%$ were diagnosed with polypoid lesions, this ensures the high probability of developing cancers from the polyps.

The low rate of participants who revealed their family history of the cancer could be due to the lack of knowledge about the disease and the embarrassing factor-stigma that leads them to hide the history of the disease.

The significant statistical association that appeared in regard to the relation of family history with the geographic distributions is rising up the importance of mode of cancer in different ethnic groups and the increasing need for a wide range of studies to reveal the inherited genetic factor of the disease.

In conclusion the endoscopic findings revealed its ability to indicate the colorectal mucosal abnormality, as it was significantly associated with the histopathological reports and showed matching results.

We concluded that colorectal lesions were common in males more than the females. And the lake of medical services could be the cause of late detection as $60 \%$ of patients were residence out of Khartoum state. It is also showed that the inflammatory bowel diseases are uncommon within the Sudanese population, and 
the western tribes are more common to have tumor or polypoid lesions although the central tribes are more common to have inflammations.

The rectum is the most common site of cancer within the Sudanese population than other parts of the colon with apparently high incidence within young ages.

\section{Acknowledgments}

special grateful thanks goes to the team of surgeon and physicians and nurses in the GIT endoscopic units of ALNeelain Medical Diagnostic Center and the staff at the GIT endoscopic units of Khartoum Teaching Hospital and Ibn sina hospital and for the staff of Histopathology lab at Ibn sina hospital, prof. Abd elsatir lab, and the histopathology lab at Khartoum hospital, Alrahma lab, and Zain lab. And a great thanks for Dr. Ibrahim Khider for helping in the completion of this manuscript.

\section{References}

1. Gimeno García AZ. Factors Influencing Colorectal Cancer Screening Participation. Gastroenterology Research and Practice. 2012;2012:1-8. https://doi. org/10.1155/2012/483417

2. Ferlay J, Shin H, Bray F, Forman D, Mathers C, Parkin DM. Estimates of worldwide burden of cancer in 2008: GLOBOCAN 2008. International Journal of Cancer. 2010 06 17;127(12):2893-2917. https://doi.org/10.1002/ijc.25516

3. Wilmink A. Overview of the epidemiology of colorectal cancer. Diseases of the colon \& rectum. 1997;40(4):483-93.

4. Saeed IE, Weng H, Mohamed KH, Mohammed SI. Cancer incidence in Khartoum, Sudan: first results from the Cancer Registry, 2009-2010. Cancer Medicine. 201405 13;3(4):1075-1084. https://doi.org/10.1002/cam4.254

5. Mohammed M eMA, Eltayeb E. Colorectal carcinoma in Sudanese patients. International Journal of Medicine. 2015〉;3(2):98-102.

6. Fund WCR, Research AIfC. Food, nutrition, physical activity, and the prevention of cancer: a global perspective: Amer Inst for Cancer Research; 2007.

7. Haggar F, Boushey R. Colorectal Cancer Epidemiology: Incidence, Mortality, Survival, and Risk Factors. Clinics in Colon and Rectal Surgery. 2009 Nov;22(04):191-197. https://doi.org/10.1055/s-0029-1242458

8. Ries LA, Harkins D, Krapcho M, Mariotto A, Miller B, Feuer EJ, et al. SEER cancer statistics review, 1975-2003. 2006...

9. O'Connell JB, Maggard MA, Livingston EH, Yo CK. Colorectal cancer in the young. The American Journal of Surgery. 2004 03;187(3):343-348. https://doi.org/10.1016/j. amjsurg.2003.12.020

10. Fairley TL, Cardinez CJ, Martin J, Alley L, Friedman C, Edwards B, Jamison P. Colorectal cancer in U.S. adults younger than 50 years of age, 1998-2001. Cancer. 2006;107(S5):1153-1161. https://doi.org/10.1002/ cncr.22012

11. O’Connell JB, Maggard MA, Liu JH, Etzioni DA, Livingston EH, Ko CY. Do Young Colon Cancer Patients Have Worse Outcomes?. World Journal of Surgery. 2004 05 06;28(6):558-562. https://doi.org/10.1007/s00268-0047306-7

12. O'Connell JB, Maggard MA, Liu JH, Etzioni DA. Rates of colon and rectal cancers are increasing in young adults. The American surgeon. 2003;69(10):866.
13. Jenkins TD, Rustgi AK. Genetics of colorectal carcinoma. cancer of the lower gastrointestinal tract Ed Willet CG London. 2001:33-44.

14. Mohamed O, Ahmed A, Roa S. Pattern \& presentation of colorectal cancer in central Sudan, a retrospective descriptive study, 2010-2012. African Health Sciences. 201505 28;15(2):576. https://doi.org/10.4314/ahs.v15i2.33

15. Abdalla AA, Musa MT, Khair R. Presentation of Colorectal Cancer in Khartoum Teaching Hospital. Sudan Journal of Medical Sciences. 200802 12;2(4). https://doi.org/10.4314/ sjms.v2i4.38498

\section{(c) (i) (-)}

This work is licensed under a Creative Commons AttributionNon Commercial 4.0 International License. 